\title{
Preparing for the Rise in Alzheimers Disease Cases: A Proposal for Training Support Personnel
}

\author{
Mark D Miller $^{1^{*}}$ and Richard K Morycz ${ }^{2}$ \\ ${ }^{1}$ Department of Behavioral Medicine, West Virginia University, Morgantown, WV, USA \\ ${ }^{2}$ Department of Psychiatry, Medicine, and Social Work at the University of Pittsburgh, USA
}

*Corresponding author: Mark D. Miller, Department of Behavioral Medicine, West Virginia University, Morgantown, WV, USA, Tel: 412-293-5112; E-mail: mark.miller1@hsc.wvu.edu

Rec date: Nov 22, 2014; Acc date: Jan 25, 2015; Pub date: Jan 27, 2015

Copyright: ( 2015 Miller MD, et al. This is an open-access article distributed under the terms of the Creative Commons Attribution License, which permits unrestricted use, distribution, and reproduction in any medium, provided the original author and source are credited.

\section{Introduction}

As the baby-boomer generation continues to age, the number of Alzheimer's disease cases is expected to double in the next decade. The functional disability and need for clinical management associated with this trend will outstrip our current workforce. Making the correct diagnosis and prescribing cognitive enhancing drugs is relatively easy compared to the time and effort required to educate and support caregivers and help them cope with the complications of neurocognitive disorders such as depression, psychosis, agitation, and growing dependency for basic function [1]. Ninety five percent of these patients will be managed in home settings that will require time and effort from family members who are traditionally women that manage their own family with children as well as their aging parents, many of whom also work themselves to make ends meet financially. Social service agencies will therefore figure large in this equation to provide day care and in-home help.

The traditional roles that social workers offer with aging adults (such as evaluating environment and functioning, assessing psychosocial support needs and how to fill them, exploring in-home assistance or alternate housing or placement options, as well as providing direct psychotherapy) are now needed more than ever [2]. Social workers are typically found playing these roles in hospitals, on inpatient psychiatry units, in multi-disciplinary geriatric care clinics, nursing homes and community-based social agencies that cater to the needs of elders.

We propose a new training strategy to train "Geriatric Care Specialists" who will be equipped with ample knowledge of gerontology, neurocognitive disorders, and depression as well as skills in managing caregiver burden dilemmas such that they can work as team members in primary care settings where most of these patients will be presenting [3]. As a geriatric psychiatrist and a social worker who have worked collaboratively for 25 years in a geriatric multidisciplinary clinic as well as educators in our respective fields, we have been training masters level social work students in a modality called Interpersonal Psychotherapy that has been modified for cognitive impairment (IPT-ci - developed at the University of Pittsburgh and field testing in the Benedum Geriatic Center at the University of Pittsburgh Medical Center).

In our experience in helping older persons with neurocognitive difficulties, we found it essential to include family caregivers as part of the social history, functional assessment, and treatment process. We discovered the importance of the timing and the order of key interventions to caregiving families. These included providing education, problem-solving strategies, and support. As we adapted traditional IPT to IPT for cognitive impairment (IPT-ci), the treatment focus embraced patient-family caregiver dyads instead of a focus on the just the problems of the identified patient. Caregivers became integrated into the delivery of care from the first contact. The IPT-ci approach became not only a treatment perspective but also a model for ongoing clinical engagement. For example, before specifically utilizing IPT-ci as a modality, we would meet with a patient, ask permission from the patient to talk the family separately to gain their perspective, and often schedule separate family psychotherapy appointments without the patient to better address caregiving issues. The IPT-ci model includes the flexibility to provide interventions (education, problem-solving, support) to family caregivers in separate or in joint meetings. We have found IPT-ci to be not only an efficient therapy, but also one that fosters understanding, empathy, and compassion for family members who are often burdened by caregiving demands and responsibilities [4]. This model offers a framework to better deliver and tailor evidenced-based interventions to burdened family caregivers, as well as advocating for the needs of the identified patient.

Interpersonal psychotherapy was originally developed by Klerman et al as pragmatic, short term psychotherapy for depression that solicits a complete picture of the interpersonal sphere of the identified patient and asks what role key relationships have played in both the onset of the depression and conversely, how the patient can renegotiate key relationships to bring symptom relief. Traditional IPT has been shown to be effective with adults, adolescents, and a variety of other populations including elders. Depression in elders typically stems from grief, role disputes with spouses, adult children or coworkers; or the many role transitions that are common in later life such as adjusting to retirement, downsizing, children moving away, and losses of sensory acumen, and robust physical health and function [5]. Loss of cognitive abilities can also be a profound role transition as patients become aware of memory loss or are forced to cease participation is activities they can no longer perform such as driving safely or complex hobbies. The approach to patients with cognitive impairment is more similar than different from an elder with depression and, of course, one commonly sees elements of both in the same patient [6]. Evaluating cognitive impairment or depression both require a thorough evaluation to make the correct diagnosis, a search for medical maladies that may have precipitated the problem (such as a thyroid disorder), appropriate diagnostic tests (which may include blood work, brain imaging and neuropsychological testing), administering appropriate medication (for depression, anxiety, psychosis, or cognitive enhancement), and, finally, providing appropriate psychosocial support [7]. This support may include specific psychotherapy interventions to resolve role disputes with caregivers, to argue for higher levels of care when indicated, and to improve coping ability for panoply of other issues that might arise in a 
Citation: Miller MD, Morycz RK (2015) Preparing for the Rise in Alzheimers Disease Cases: A Proposal for Training Support Personnel. J

given older patient. Using either traditional IPT for non-demented elderly patients needs or IPT-ci for those with cognitive disorders is an ideal format for teaching a practical approach to managing the range of needs of older patients. IPT was designed to work seamlessly in consort with pharmacotherapies; thus social workers trained in IPT/ IPT-ci are ideal candidates for the "Geriatric Care Specialist" position we mentioned earlier.

The efficacy of IPT-ci has not been tested to date in a randomized controlled trial; however, we offer the following small sample of descriptive data that we argue supports its face validity. We utilized IPT-ci in a quality improvement project at the Benedum Geriatric Center at the University of Pittsburgh a few years ago by applying the approach to all new admissions to our multidisciplinary geriatric care clinic over a 6 month period and tracked their progress for a year [8]. An analysis of the first 15 patients showed them to have cognitive function ranging from scores of 12-28 on the Montreal Cognitive Assessment Screening tool and PHQ-9 scores from 3-22 at baseline. All but two subjects were accompanied by caregivers, six of whom were spouses and the rest were adult children The IPT focus was role transition to a less functional state in 14 subjects, and interpersonal deficit in one. Ten subjects showed a significant burden of depression with a baseline PHQ-9 of 10 or greater with a mean scores of 17.6 at baseline and repeat testing showed PHQ- 9 scores of 10.2 at 3 months, 9.1 at 6 months and 7.5 at 12 months [9]. Nonparametric statistical comparison of change from baseline to 3 months: $n=9, p=0.0625$; to 6 months, $\mathrm{n}=9, \mathrm{p}=0.0078$; to 12 months, $\mathrm{n}=7, \mathrm{p}=0.0313$ [10]. These data show reduction in depression severity scores over time compared to baseline although they must be interpreted with caution as the sample size is very small, patients were seen at variable follow-up intervals, no independent raters were used for repeat measures, there was no attempt to control for the effects of any psychotropic medication use, and there is no control group for comparison. As a quality improvement project using this small case series, the IPT-ci approach delivered in the context of a multidisciplinary geriatric treatment center did appear to contribute to a reduction in depression severity despite further progressive cognitive decline in some subjects [11]. As a result, we have subsequently incorporated these techniques into our standard practice at the Benedum Geriatric Center [12].

In conclusion, our combined 60 years of experience collaborating on the treatment of mental illness in elders (as a geriatric psychiatrist and a geriatric social worker) have served as a background for developing the principles behind the adaptation of IPT for depressed or cognitively impaired elders and their caregivers [13]. We urge other institutions of higher learning to consider incorporating the principles of IPT/IPT-ci into their curriculums as a practical and efficient way to help train the army of compassionate geriatric mental health specialists we are going to need very soon.

\section{References}

1. Ganguli M, Rodriguez EG, Mulsant B, Richards S, Pandav R, et al. (2004) Detection and management of cognitive impairment in primary care: the Steel Valley Seniors Survey. Journal of American Geriatrics Society 52: 1668-1675.

2. Klerman GL, Weissman MM, Rounsaville BJ (1984) Interpersonal psychotherapy of depression. NewYork, Basic Books.

3. Klerman G, Weissman MM (1993) New Applications of Interpersonal Psychotherapy. Washington, DC, American Psychiatric Press.

4. Kroenke K, Spitzer RL, Williams JB (2001) The PHQ-9: validity of a brief depression severity measure. Journal of General Internal Medicine 16: 606-613.

5. Miller MD (2004) Using Interpersonal Psychotherapy for depressed elders with cognitive impairment. The Clinical View. Geriatric Psychiatric in Long-Term Care 2.

6. Miller MD, Reynolds CF (2007) Expanding the usefulness of Interpersonal Psychotherapy (IPT) for depressed elders with co-morbid cognitive impairment. International Journal of Geriatric Psychiatry 22: 101-105.

7. Miller MD (2009) Clinicians Guide to Interpersonal Psychotherapy in Late life; Helping Cognitively Impaired or Depressed Elders and their Caregivers; Oxford University Press.

8. Miller MD, Morse J (2012) Psychotherapy in Late-Life. In Pittsburgh Pocket Psychiatry Series: Geriatric Psychiatry. Miller MD and Solai L. Eds., Oxford University Press.

9. Miller MD, Reynolds CF (2012) Using IPT in Older Individuals. In Casebook of Interpersonal Psychotherapy. Markowitz, JC, Weissman, MM (Eds.), Oxford University Press, New York.

10. Morycz RK (1985) Caregiving strain and the desire to institutionalize family members with Alzheimer's disease. Research on Aging, 7: 329-361.

11. Morycz RK, Malloy JE, Bozich MA (1987) Racial differences in family burden: Clinical implications for social work. Journal of Gerontological Social Work, 10: 133-154.

12. Nasreddine ZS (2004) The Montreal Cognitive Assessment (MoCA): a Brief Cognitive Screening Tool for Detection of Mild Cognitive Impairment. Neurology, Volume 62, Number 7 S(5) April 2004, A132. Presented at the American Academy of Neurology Meeting, San Francisco.

13. Schulz R, Morycz R (2012) Sociological Context of Late Life. In Pittsburgh Pocket Psychiatry Series: Geriatric Psychiatry. Miller MD, Solai L(eds.) Oxford University Press. 